\section{Espacios que educan: tres momentos en la historia de la educación en Argentina}

Sonia Sasiain *

Resumen: El objetivo de este trabajo es analizar tres momentos en la historia de la educación pública en Argentina en los que hubo un cambio en las formas de entender al alumno y a su contexto educativo como una experiencia transformadora.

La identidad nacional está conformada por las instituciones, y la educación, desde la escuela hasta la universidad, tiene un lugar central en esta construcción.

1. Desde que Argentina declaró su independencia, y sobre todo cuando Buenos Aires se convirtió en la capital de la nueva república en 1880, un vertiginoso proceso de modernización se inició. Este proyecto involucró a los inmigrantes que llegaron de diferentes países a la capital y la transformaron en una Babel que el gobierno tuvo que "argentinizar". Así, la educación pública obligatoria, gratuita y laica fue reglamentada en 1884 con la aprobación de la Ley 1420. Las escuelas públicas proveyeron el nuevo espacio donde los estudiantes pudieron obtener los valores nacionales.

2. Durante el primer peronismo (1943-1955) la educación técnica tuvo un papel central dentro del proyecto modernizador que en el discurso gubernamental se presentaba como revolucionario: el de la Nueva Argentina, donde los trabajadores ocuparon un lugar privilegiado. En este período se crearon diversas tipologías: desde la escuela-fábrica hasta la Universidad de los Trabajadores en la que, a través de un nuevo sistema de administración, el espacio escolar ofrecía servicios a la comunidad que superaban la función educativa tradicional.

3. En la actualidad, el sitio donde había una antigua bodega, en una de las zonas más cotizadas de Buenos Aires, ha sido reciclado para desarrollar un proyecto educativo: el Polo científico y tecnológico, dividido en diferentes secciones: la sede del Ministerio de Ciencia, Tecnología e Innovación Productiva, CONICET y la Agencia Nacional de Promoción de Ciencia y Tecnología e institutos de investigación interdisciplinaria, con una conexión internacional a gran escala. El nuevo complejo está diseñado como un centro de gestión, producción y difusión del conocimiento.

Palabras clave: arquitectura - educación - espacio - identidad - modernidad.

[Resúmenes en inglés y portugués en las páginas 299-300]

${ }^{(*)}$ Licenciada en Artes. Egresada del Programa de Preservación y Conservación del Patrimonio de la Universidad Torcuato Di Tella, donde en la actualidad cursa la Maestría de Historia y Cultura de la Arquitectura y de la Ciudad. Dicta clases en su especialidad en la Universidad Torcuato Di Tella, en la Facultad de Arquitectura y de Urbanismo, UBA y en el Instituto Universitario Nacional del Arte. 
El maestro no inventa la ciencia, ni la enseña, acaso la alcanza sino en sus más simples rudimentos: acaso la ignora en la magnitud de su conjunto: pero él abre las puertas cerradas al hombre naciente y le muestra el camino; él pone en relación al que recibe sus lecciones con todo el mundo, con todos los siglos, con todas las naciones; con todo el caudal de conocimientos que ha atesorado la humanidad. (...) Enseñemos a leer esos caminos de hierro, esos telégrafos eléctricos, esos vapores que como las obras de la naturaleza narran la gloria de Dios; así ellos van narrando por todos los países de la tierra la gloria y el poder de las naciones que han cultivado la inteligencia y prodigando los medios de conocer y participar del caudal de luces que ha atesorado la humanidad. (Sarmiento, Santiago de Chile, octubre de 1852, Publicado en Revista La Educación)

\section{Introducción}

Desde que se sancionó el proyecto de educación pública en 1884, las escuelas proveyeron el nuevo espacio donde los estudiantes pudieron internalizar los valores nacionales. Valores que fueron adecuándose a los cambiantes vaivenes de la economía y de los modelos educativos que, en cada momento, los dirigentes consideraban adecuados al proyecto de país. En este trabajo, la perspectiva que se ha elegido para abordar la relación entre espacio escolar y educación es la de analizar el rol que jugó la enseñanza técnica en tres períodos, sin pretender agotar la imbricada trama de fuerzas y conflictos existentes en cada momento. Cada etapa implicó búsquedas, exploración, de distintas configuraciones tipológicas y funcionales apropiadas para esos edificios. Los ejemplos estudiados servirán como disparadores para provocar una reflexión acerca de los ámbitos educativos como facilitadores o no de la adquisición de una cultura técnica y de los límites que un proyecto de país sin educación técnica enfrenta.

En 1884, el Estado consideraba a las escuelas entre sus edificios más representativos, que daban clara cuenta de la nación moderna en la que se estaba convirtiendo, llamada a ocupar un lugar destacado entre los países industrializados del mundo.

En la actualidad, en un panorama de crisis de las identidades políticas y económicas tradicionales, el papel de la educación en la sociedad y, más específicamente, en la formación del ciudadano, se encuentra en constante redefinición. Las certezas que dieron origen el proyecto educativo público del s. XIX se han perdido y, en un mundo donde la información y los conocimientos se acumulan y circulan a través de medios tecnológicos cada vez más sofisticados y poderosos, la arquitectura escolar también debe dar respuesta a estos desafíos.

\section{El nacimiento del proyecto educativo}

Desde 1880 el país emprende, a un ritmo acelerado, un proyecto modernizador después de un largo período de luchas internas, y desarrolla una política claramente favorable a la inmigración. Alrededor del 90\% de los migrantes netos del período 1857-1914 se estableció 
en las ciudades de la región pampeana, preferentemente en Buenos Aires, contribuyendo a un temprano desarrollo de la economía de servicios y a una incipiente actividad industrial. En el período 1880-1930 se produjo una vertiginosa transformación económica y social. Se inició la creación de una nación moderna a través de tres áreas principales: educación universal, inmigración y atracción de capital y tecnología extranjera. El Estado pronto quiso "argentinizar" a los recién llegados y así surgió la educación pública obligatoria, laica y gratuita en 1884 con la sanción de la ley 1420. En este contexto, la arquitectura de los espacios escolares ocupó un lugar central en los debates.

Nuestras escuelas deben (...) ser construidas de manera que su espectáculo, obrando diariamente sobre el espíritu de los niños, eduque su gusto, su físico y sus inclinaciones. No sólo debe reinar en ellas el más prolijo y constante aseo (...) sino también tal comodidad para los niños y cierto gusto y aún lujo de decoración, que habitúe sus sentidos a vivir en medio de estos elementos indispensables de la vida civilizada (Sarmiento, 1949: 235).

Los alumnos adquirirían los valores nacionales al atravesar la puerta de la escuela a través de la práctica de una nueva cultura en sus espacios: maniobras militares en los patios -como entrenamiento ante la posibilidad de un conflicto bélico-, la conmemoración de los 'héroes de la patria' en los salones de actos, y las clases en la lengua oficial en las aulas de las nuevas escuelas públicas. En este contexto, se dio comienzo a la construcción de los nuevos edificios, fundamentalmente, para la enseñanza primaria.

\section{La educación popular argentina}

Domingo Faustino Sarmiento maestro rural, ensayista y político, presidente de la nación durante el período 1868-1874, fue uno de los principales promotores de la educación pública en el país. En La educación popular (1949) hizo un análisis de tratados de arquitectura escolar de Estados Unidos y de varios países europeos, además de observaciones directas que realizó durante sus viajes. Insistió en prestar mucha atención a las cuestiones de higiene asoleamiento y ventilación de los edificios escolares. La primera escuela primaria construida en 1858 para tal fin, inspirada en las ideas sarmientinas fue la de Catedral al Norte, en la Capital Federal, proyectada por el arquitecto Miguel Barabino. Austera, con una fachada de inspiración neorrenacentista daba cuenta de los problemas que presentaba la magra asignación del Estado nacional en las escuelas primarias que, en su mayoría -se abrieron ochocientas durante su presidencia-, funcionaban en edificios preexistentes, mal ventiladas y sin condiciones higiénicas adecuadas.

Sarmiento confiaba en la autogestión de los ciudadanos para financiar los edificios escolares como parte de un proyecto pedagógico que iba ligado al de la explotación de industrias en escalas intensivas. Su proyecto educativo estaba en total sintonía con los debates de la época acerca del modelo económico que el país debía adoptar: industrial o, el que finalmente ganó: agroexportador. Sarmiento proponía crear escuelas técnicas ligadas a las economías regionales, según el avance del desarrollo técnico de su época ya que consideraba 
que el país no contaba con las condiciones naturales que sí habían favorecido a Inglaterra: la abundancia de carbón y la cercanía a los sistemas de comunicación fluvial. La prueba de ello es que fomentó la creación de la Escuela de Minas de San Juan y la Escuela de Vitivinicultura en Mendoza, además de las escuelas normales para la formación de los futuros maestros. Para Sarmiento el país adquiriría un lugar en el concierto de las naciones a través de la modernización por la industrialización. En su proyecto, la educación popular ocupaba un lugar central, e imaginaba que los egresados de las escuelas estarían preparados para el comercio y para la agricultura intensiva. Las escuelas, para Sarmiento, no debían enseñar un oficio, sino los principios científicos de cuya aplicación dependen esos oficios. Su propuesta no prosperó en los dos aspectos, las escuelas crecieron a un ritmo sostenido recién después de 1884, no por la financiación privada sino cuando el Estado comenzó a asignar partidas presupuestarias para su construcción, y el modelo económico dominante no promovió la creación de escuelas técnicas e industriales en la escala que Sarmiento esperaba, sino que promovió las dedicadas a la enseñanza humanística. En definitiva, la integración del país en la división internacional del trabajo como proveedor de bienes primarios y la adopción del modelo agroexportador en detrimento del proyecto industrializador sarmientino, definió el contenido curricular educativo y los espacios necesarios para desarrollarlo.

\section{En busca de la forma}

Una vez sancionada la Ley 1420 y en el contexto de construcción de los edificios representativos de la nación se dio una amplia experimentación tipológica que buscó brindar las unidades educativas suficientes para cumplir con la oferta de educación obligatoria y gratuita y, a la vez, utilizarlos como emblemas de expansión de los ideales civilizatorios del Estado.

Una de las tipologías más notables, desarrolladas en esta época, es el de las escuelas-palacio ejemplo es el de la escuela Petronila Rodríguez (1882-1895), [Ver Figura 1 en Catálogo de Figuras en página 296] actual sede del Ministerio de Educación, de influencia germánica, diseñada por los arquitectos Carlos y Hans Altgelt. Las dimensiones de esta construcción y el carácter de su diseño y decoración expresaban las búsquedas, dentro del eclecticismo decimonónico, del estilo nacional que representara al Estado moderno argentino. La composición académica de ésta y de otras escuelas-palacio implicaba una organización jerárquica de los espacios y del equipamiento destinado a satisfacer las distintas funciones: administración y dirección, aulas, talleres, laboratorios, museo de historia natural, biblioteca; todo amoblado con pupitres, escritorios y variado material didáctico especialmente diseñado. Los nuevos criterios pedagógicos y funcionales se acompañaban de un notable despliegue formal en fachadas, vestíbulos, auditorios y bibliotecas.

Para entender la importancia civilizatoria de estos edificios, baste considerar que en 1887 el poder ejecutivo decidió emplazar al Palacio del Congreso Nacional en la manzana situada entre las calles Callao, Paraguay, Rodríguez Peña y Charcas, que enfrentaba la ya avanzada construcción de la escuela Petronila Rodríguez. De haber prosperado esta iniciativa, 
una vez terminados los dos edificios, dialogarían como símbolos de la moderna nación. Para ello, se encomendó al arquitecto Francesco Tamburini (autor del diseño exterior a la Casa Rosada) un proyecto que mostraba la clara influencia del edificio escolar en su propuesta para el edificio legislativo que, finalmente, se construyó en su ubicación actual: Callao y Rivadavia (Shmidt, 2012).

La enseñanza graduada, acorde a las edades de los alumnos, disponía distintos espacios en respuesta al programa de necesidades según el nivel escolar: inicial a universitario, y al tipo de actividades que en ellos se desarrollaba. La enseñanza secundaria, optativa, se cursaba en escuelas normales, en las que se formaban los futuros maestros, y en colegios nacionales, que preparaban a los estudiantes para completar estudios en profesiones liberales. En 1890 surgen los colegios comerciales, y en 1897 se funda el primer colegio secundario industrial de la Nación que fue creado como Departamento Industrial, anexo a la Escuela Superior de Comercio, en respuesta a la creciente necesidad de enseñar temas relacionados con la ciencia aplicada y con la técnica. Fue el origen de la Escuela Industrial de la Nación, que dirigió el ingeniero Otto Krause en 1897.

Inspirada en las escuelas técnicas de artes y oficios europeas, contó originalmente con tres especialidades: Química, Construcciones y Mecánica y más tarde incorporó Electrotécnica. En 1903 se proyectó un edificio propio que constaba de sectores para Museo Tecnológico, Sala de Ciencias Naturales, Sala de Máquinas y Sala de Construcciones. En 1909 se inauguró esta escuela de inspiración germánica que, detrás de una fachada ornamentada con detalles por detalles constructivos, combina ladrillo, hierro y símil piedra. En el interior, los talleres se desarrollan en espacios de aspecto industrial. En 1910 asistían a esos establecimientos unos 5600 alumnos de Buenos Aires y del interior del país.

En el promedio de las escuelas públicas secundarias construidas, priman las destinadas a la enseñanza humanística sobre las industriales o técnicas hasta 1945. La enseñanza denominada en ese período "especial", destinada a instruir y a preparar a los jóvenes con un oficio con salida laboral, estuvo en general promovida por la iniciativa privada, por fuera de las acciones oficiales. En Buenos Aires, en 1900, la Sociedad de Educación Industrial desarrolló un programa de educación para el trabajo técnico en sus distintas manifestaciones. Hasta 1914 se abrieron escuelas en las que predominó un curriculum adecuado para una industria básica: mecánicos, electricistas, escuelas nocturnas de dibujo, chauffeurs, plástica ornamental, dibujo para niñas, radiotelegrafía, química e industrial y construcciones y resistencia de materiales. Para albergar estas escuelas, la Sociedad construyó una serie de pabellones y talleres dentro de una arquitectura inspirada en el clasicismo francés del siglo XVIII. Estos edificios, en el barrio de Almagro, en la Capital Federal, en la actualidad, son sede de la Universidad Tecnológica Nacional.

El menor desarrollo de la enseñanza técnica e industrial, en relación con otras especialidades, en esta etapa temprana, se debe a que, hasta fines del siglo XIX, la nación se mantuvo en el sistema mundial como proveedora de alimentos y materias primas y fue receptora de capital, tecnología y fuerza de trabajo. Esto determinó que, hasta 1940, la mano de obra necesaria para trabajar en la industria, no fuera calificada y, por lo tanto, estuviera en condiciones de integrarse al trabajo con una educación elemental. 


\section{Sustitución de importaciones y enseñanza técnica}

A partir de 1930 se da en nuestro país el modelo de sustitución de importaciones que implica el crecimiento de las ciudades cercanas a la zona portuaria del Río de la Plata en las que se radican fábricas y se hace necesario contar con escuelas para educar a los hijos de los obreros.

Tanto la alta burguesía agraria como la burguesía industrial, conectada con el capital extranjero que fijaba los límites del desarrollo local, consideraban que un proyecto industrial que superara la sustitución de importaciones amenazaba su hegemonía. En este contexto, la enseñanza técnica debía cumplir con un doble propósito: formar a un sector de los alumnos provenientes de los estratos más bajos en las Escuelas de Artes y Oficios para su inmediata inserción en el mundo laboral o bien, preparar a los provenientes de los sectores medios y altos para continuar estudios universitarios afines a través de la formación en la Escuela Industrial.

\section{La incorporación de la clase obrera al sistema educativo}

El rol periférico que ocupaba la Argentina una vez lanzada la carrera por la industrialización estableció una brecha que no se podía sortear sin decisión política, sobre todo en el campo educativo. Entre 1943 y 1955 se abrió un nuevo período dentro de la enseñanza técnica, que adelantaba el inicio al nivel primario y culminaba en la universidad. Se amplió el viejo sistema y se crearon nuevas alternativas. Sin duda, el cambio de orientación de los estudios técnicos coincidió con el agotamiento del proceso 'fácil' de sustitución de importaciones y la introducción progresiva de cambios tecnológicos que determinaron necesidades de calificación muy diferentes de las derivadas de la manufactura. En términos del trabajo, el paso de la manufactura a la mecanización y a la automatización produjo el crecimiento de técnicos profesionales que requirieron calificaciones formales de larga duración. Durante el primer gobierno peronista, el Estado promovió profundos cambios en los contenidos y en la escala de la planificación escolar. Como parte de un proyecto industrializador, se lanzó el Primer Plan Quinquenal, que en lo educativo incluía un desarrollo masivo y abarcaba a todos los niveles de la enseñanza técnica.

Pablo Pineau (1991) demuestra que en nuestro país, el modo en que fue concebido y valorizado el trabajo durante el peronismo, marcó una diferencia esencial con el tradicional modelo educativo al incorporar a los trabajadores en el nivel de las decisiones y en la planificación escolar, porque el objetivo era producir, en la estructura política, un cambio capaz de modificar la relación tradicional entre saber y trabajo. El cambio de modelo educativo partía del nivel primario, incluía el nivel medio (escuelas-fábricas, escuelas industriales de la Nación) y llegaba hasta el nivel universitario con la Universidad Obrera Nacional (UON), y gozaba, en su totalidad, de un alto grado de autonomía respecto del sistema tradicional. La posibilidad de sostener la enseñanza técnica implicaba la promoción educacional de sectores hasta entonces marginados (clase obrera y demás sectores populares) [Ver Figura 2 en Catálogo de Figuras en página 296]. 
La inclusión de estos sectores suponía que el Estado aseguraría las condiciones de vida que les permitieran asumir el costo de los estudios y, a los hijos de los obreros, postergar su ingreso a la actividad productiva. Las autoridades plantearon un nuevo sesgo pedagógico y otra concepción de la escuela y de la comunidad en la que estaba inserta. Se incorporaron al programa de necesidades del edificio escolar salas de espectáculos, museos, gimnasios, con ánimo de extender su uso a la población; y en los halls de ingreso, esculturas y murales de carácter nacional que ampliaron sus funciones a contra-turno. “... la escuela no puede ni debe circunscribir su acción a lo meramente formativo en cuanto al alumno, sino que asimismo, ha de cumplir la misión de un verdadero centro cultural dentro de su esfera..." (Actas de las III Jornadas de Investigación de la FAU, 2 y 3 de noviembre de 2006).

Entre utopías y realizaciones, la década de 1950 se inició con notables innovaciones en materia arquitectónica. Desde el Ministerio de Obras Públicas se diseñaron escuelas de acuerdo a los parámetros del "estilo internacional" que implicaban seguir los postulados funcionalistas característicos de esta arquitectura. "Era el tiempo de los revestimientos hechos con vidrio molido, pequeños mosaicos, ladrillos de piedra, cerámicos coloreados, pequeños listones de madera, linóleo o goma" (Grementieri y Shmidt 2010, p. 210). Una imagen de líneas netas, inspirada en la tipología industrial dará lugar a una renovación en la arquitectura escolar que por más de veinticinco años guiará experimentos y realizaciones hacia el futuro.

En 1944 se había creado la Comisión Nacional de Aprendizaje y Orientación Profesional (CNAOP) que durante mucho tiempo estuvo bajo el control directo del Ministerio de Trabajo, y no del de Educación, lo que la convertía en una instancia más flexible ante los requerimientos del nuevo orden político: regulaba el aprendizaje industrial y el trabajo de menores (horarios, condiciones, seguridad, etc.) En el gobierno de la CNAOP se encontraban representantes de los sectores industriales y de los obreros. Hacia 1948 se procuró unificar, bajo una misma dirección, la gestión de las escuelas industriales de la Nación, las de agronomía, las profesionales de mujeres y las de artes y oficios.

Es razonable que, como todo grupo social en ascenso, este sector pretendiera contar con una instancia superior para completar sus estudios, lo que determinó la creación de la UON (Universidad Obrera Nacional), destinada a permitir el paso de los egresados de los establecimientos de la CNAOP al nivel universitario. La universidad se organizaba a través de facultades obreras regionales. En Córdoba, entre otras especialidades, se ofrecía "Construcciones Aeronáuticas"; en Mendoza, "Construcciones de Obras Antisísmicas”, y en Tucumán, "Industrias Químicas" y "Construcciones Ferroviarias".

El gobierno de la universidad era ejercido por el rector, que debía ser un egresado de la escuela sindical dependiente de la (CGT) Confederación General del Trabajo. Estaba asesorado por un Consejo de Coordinación Industrial, con participación patronal y obrera. Después del golpe de estado de 1955, se terminó con el proyecto educativo del peronismo, la UON, creada en 1948 como la cúpula del sistema, con fines de investigación, promoción y asesoramiento a la industria. En 1959 se transformó en la Universidad Tecnológica Nacional. 


\section{Los desafíos actuales}

Después de 2001, tanto a nivel local como internacional, hay consenso en los discursos que reconocen un profundo proceso de transformación. "'Sociedad de la información', 'sociedad poscapitalista,' 'sociedad post-industrial', 'nueva edad media,' 'tercera ola', son algunas de las expresiones que diversos autores como Jacques Delors, Peter Drucker, Alain Minc o Alvin Toffler han popularizado en los últimos años" (Tedesco,1996).

Frente a las nuevas formas de organización social, económica y política existentes, y a la extrema complejidad que han alcanzado la técnica y la ciencia en la actualidad, ya no es posible pensar a la escuela como la institución socializadora y difusora del conocimiento tal como se la concibió en 1884. El valor concedido al conocimiento, especialmente al tecnológico, constituye la variable más importante de las nuevas formas de organización social y económica actuales. "Ya se ha transformado en un lugar común la afirmación según la cual los recursos fundamentales para la sociedad y para las personas serán la información, el conocimiento y las capacidades para producirlos y para manejarlos." (Tedesco, 1996) Esta concepción del saber demanda nuevos programas arquitectónicos para la educación que se complejizan y deben atender tanto a la producción como a la transmisión de conocimiento. Entre 1976 y 2001 se da en el país un plan sistemático de desmantelamiento del aparato productivo que tiene su correlato en los espacios educativos. Se produce el avance de teorías neoliberales en el diseño curricular que no privilegian la educación técnica o industrial y se recorta el presupuesto en investigación. Para revertir esta situación se crea en 2007 el Ministerio de Ciencia, Tecnología e Innovación Productiva. Dentro de las innovaciones y programas que se desarrollan en el área se proyecta el Polo Científico y Tecnológico, un edificio que recicla aquel en el que funcionó la antigua Bodega Giol, en una de las zonas más cotizadas de Buenos Aires. Dividido en diferentes secciones: la sede del Ministerio de Ciencia Tecnología e Innovación Productiva, el CONICET (Consejo Nacional de Investigaciones Científicas y Técnicas) y la Agencia Nacional de Promoción de Ciencia y Tecnología e institutos de investigación interdisciplinaria, con conexión internacional; el nuevo complejo está diseñado como un centro de gestión, producción y difusión del conocimiento.

\section{La arquitectura para la educación y la investigación}

El proyecto abarca $45.000 \mathrm{~m}^{2}$ y conserva las fachadas y estructuras originales de la antigua bodega que han sido puestas en valor. Para adecuar la estructura existente al nuevo programa de necesidades, se evitó la demolición de lo antiguo, lo que ha constituido una operación de ahorro de energía por sí misma; al mismo tiempo que se buscó preservar el valor histórico del patrimonio arquitectónico del barrio. También se instalaron diversos sistemas de racionalización del uso de la energía tanto en su construcción como para su mantenimiento: energía solar para el calentamiento del agua de los sanitarios, sistemas que permiten el control y ahorro de la energía eléctrica para alimentar a los equipos informáticos, a los sistemas de iluminación, de climatización y de refrigeración. Para reducir el consumo de agua, el emprendimiento incorpora un sistema que reutiliza el agua pro- 
veniente de descargas secundarias (lavatorios y cocinas) a la que se le aplica un antiespumógeno para que, luego de ser filtrada y coloreada, pueda ser reutilizada para la descarga en inodoros y mingitorios.

\section{El edificio}

En el complejo se encuentra la sede del Ministerio de Ciencia, Tecnología e Innovación Productiva de la Nación [Ver Figura 3 en Catálogo de Figuras en página 296], cuya misión, según sostienen las autoridades, es "orientar la ciencia, la tecnología y la innovación hacia el fortalecimiento de un nuevo modelo productivo que genere mayor inclusión social y mejore la competitividad de la economía argentina, bajo el paradigma del conocimiento como eje del desarrollo." Además, el edificio cuenta con un restaurante de libre acceso ubicado en la planta baja y tiene comunicación directa con una plaza pública.

En el denominado Edificio Blanco, se encuentra la Agencia Nacional de Promoción Científica y Tecnológica, organismo que financia actividades relacionadas a la ciencia, la tecnología y la innovación productiva. En este mismo sector, también funcionan los Institutos Internacionales Interdisciplinarios para la Innovación, que fomentan las relaciones internacionales en investigación y desarrollo en ciencia, tecnología e innovación productiva. Estos son: el Instituto de investigación en Biomedicina de Buenos Aires CONICET-Instituto partner de la Sociedad Max Planck en Buenos Aires: dedicado a temas actuales de las biociencias, especialmente al ámbito de la investigación en biomedicina. Además, se encuentran la Unidad de Investigación y Capacitación del ICGEB (Centro Internacional de Ingeniería Genética y Biotecnología), Centro bilateral de Diseño Industrial (Argentina- Italia), Centro Interdisciplinario de Estudios en Ciencia y de Tecnología e Innovación (CIECTI). El CONICET ocupará un espacio en un sector, actualmente en desarrollo, para realizar investigaciones en cuatro áreas: ciencias agrarias, ingeniería y de materiales; ciencias biológicas y de la salud; ciencias exactas y naturales y ciencias sociales y humanidades.

El Polo contará con un Museo Interactivo de Ciencia que albergará una exposición permanente; más un sector de Biblioteca, Mediateca, Aulas y talleres y un hall central y una cafetería. Este espacio estará abierto a todo el público, y pretende conectar a los científicos con el público en general y convertirse en un centro de difusión de la ciencia y de integración de sus conceptos con la sociedad.

Finalmente, el proyecto se completa con un Auditorio que contará con dos salas de conferencias y un salón principal apto para eventos académicos.

\section{Conclusión}

Desde sus orígenes a mediados del siglo XIX, la escuela pública buscó integrar disciplinadamente a la mayoría de la población, tradicional o recién llegada, dentro de la república en expansión. La matriz moderna que vincula proyecto de país y mundo del trabajo, ciudadanía y cultura letrada, alfabetización y soberanía popular, signó la historia de la nación 
entre fuertes conflictos y debates. La arquitectura para la educación pública confió en el poder transformador de los nuevos edificios sobre los alumnos albergados en su interior o en los ciudadanos que veían materializarse en las escuelas-palacio la avanzada civilizatoria moderna. Pero este primer proyecto encontró pronto sus límites e implicó la intervención estatal para fijar los límites del desarrollo local, sin privilegiar el proyecto industrial, lo que delineó una educación técnica acorde a una producción básica.

Tal como señala Pablo Pineau (2006) el primer peronismo fue el artífice más completo del modelo nacional en el que 'ser argentino' significaba ser alfabetizado, ser ciudadano y tener un trabajo asegurado, mediante el máximo despliegue del Estado-Nación alcanzado en Argentina, cuyos efectos de exclusión marcan, a la vez, los límites de su expansión. Se crearon nuevas tipologías como las de las escuelas-fábrica que, junto a las reformas organizativas de la enseñanza técnica, en todos los niveles educativos, acompañaron la construcción de nuevos edificios en sintonía con el estilo de la arquitectura pública del período para definir una imagen industrial en estos establecimientos como símbolo de un Estado moderno que impulsaba un nuevo modelo productivo.

En la actualidad, el proyecto del Polo Científico Tecnológico nos ha permitido reflexionar sobre algunos aspectos que resumen el desarrollo de la arquitectura para la educación pública a lo largo de más de un siglo, en el gesto de apelar a una mirada patrimonial a la vez que desarrolla espacios para investigaciones con la más avanzada tecnología. La convivencia en el mismo predio de estas dos estructuras muestra los problemas que presenta la preservación del patrimonio tangible (la antigua bodega que, reciclada, alberga al nuevo Polo Científico y Tecnológico) e intangible (la cultura de la inclusión social a través de la educación y del trabajo). Este proyecto es el comienzo de un giro hacia un modelo de desarrollo tecnológico e industrial que debería tener un correlato en lo económico y en lo político a largo plazo para que optimice los recursos públicos, materiales y humanos, acumulados a lo largo de varias generaciones.

La arquitectura hoy enfrenta el desafío de preservar, construir y equipar escuelas que permitan a los educadores preparar a los alumnos en el uso consciente, crítico, activo, de la información y del conocimiento. El Estado, a la vez, debe garantizar que sus egresados encuentren un espacio propicio para poner en práctica lo aprendido. 


\section{Bibliografía}

Grementeri, F. y Shmidt, C. (2010). Arquitectura, educación y patrimonio. Argentina 16001975. Buenos Aires: Pamplatina.

Longoni, R. et al. (2006). Aulas bonaerenses. un siglo de infraestructura escolar en la Provincia de Buenos Aires. Publicado en Actas de las III Jornadas de Investigación de la FAU, 2 y 3 de noviembre de 2006.

Pineau, P. (1991). Sindicatos, estado y educación técnica 1936-1968, Buenos Aires: Centro Editor de América Latina.

[et al.] (2006). El principio del fin. Políticas y memorias de la educación en la última dictadura militar. (1976-1983). Buenos Aires: Colihue.

Puiggros, A. (dir.) (2006). Historia de la Educación Argentina. Peronismo: Cultura política y educación (1945-1955) T.V. Buenos Aires: Galerna.

Sarmiento, D. F. (1949). La educación popular. Buenos Aires: Lautaro.

Shmidt, C. (2012). Palacios sin reyes. Arquitectura pública para la "capital permanente". Buenos Aires, 1880-1890. Rosario: Prohistoria.

Snow, C. P. (1987). Las dos culturas y un segundo enfoque. Madrid: Alianza.

Tedesco, J. C. (2003). Educación y sociedad en la Argentina (1880-1945). Buenos Aires: S. XXI. (1996). Nueva Sociedad Nro. 146 Noviembre-Diciembre 1996, pp. 74-89. Disponible en www.nuso.org. Consultado el 25/5/12.

Torrado, S. (compiladora). (2006). Población y bienestar en la Argentina del primero al segundo Centenario. Una historia social del siglo XX. Tomo II. Buenos Aires: Edhasa.

Polo Científico Tecnológico. Disponible en www.polo.mincyt.gob.ar/ Consultado el 10/3/12. 


\section{Catálogo de Figuras}

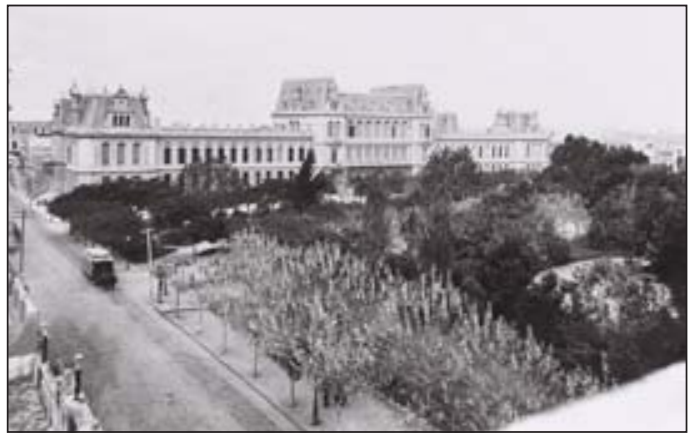

F1.

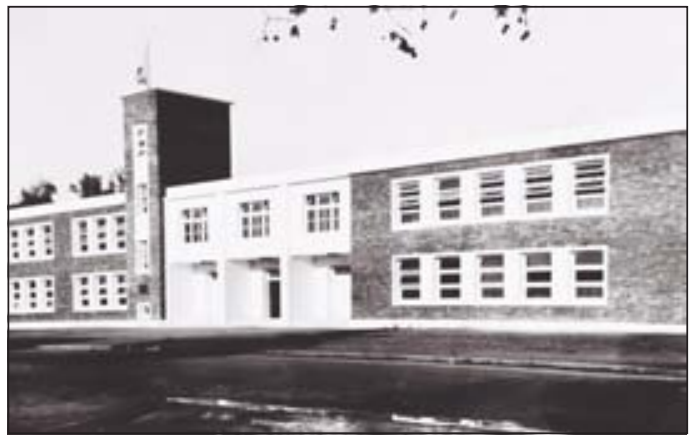

F2.

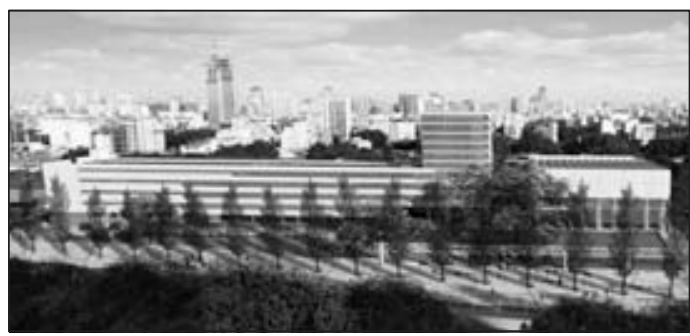

F3.

Figura 1. Escuela Petronila Rodríguez 1882-1895 actual Palacio Sarmiento sede del Minis-terio de Educación de la Nación Argentina. Fuente. Gentileza Archivo General de la Nación. Figura 2. Escuela Fábrica $\mathrm{N}^{\text {ro }} 1$ Casal Calviño, 1948. Fuente. Gentileza Archivo General de la Nación.

Figura 3. Polo Científico Tecnológico. Inauguración de la primera etapa, 2011. Fuente. www.polo.mincyt.gob.ar/ 
Summary: The aim of this paper is to analyze three moments in the history of public education in Argentina in which there was a change in the ways of understanding the student and the educational context as a transformational experience.

The national identity is shaped by institutions, and education, from school to university, has a central place in this construction.

1. Since Argentina declared its independence, and especially when Buenos Aires became the capital of the new republic in 1880, a vertiginous modernization process began. This project involved the immigrants who arrived from many different countries to the capital and transformed it into a Babel that the government had to 'Argentinize'. Thus the compulsory public education, free and secular was declared in 1884 with the approval of Law 1420 . The new public schools provided the spaces where students could obtain national values. 2. During the first Peronism (1943-1955) technical education had a central role in the modernization project that, in the government's discourse, is presented as revolutionary: that of the Nueva Argentina where workers occupied a privileged place. By this period many typologies were created: from factory-schools to Workers' University in which, through a new system of administration, the school space provided services to the community that exceeded the educational traditional role.

3. Today, the site formerly a winery in one of the most desirable areas in Buenos Aires, has been recycled to develop an educational project: the scientific and technological center, divided into different sections: the headquarters of the Ministry Science, Technology and Productive Innovation, CONICET and the National Agency for Promotion of Science and Technology and interdisciplinary research institutes, with a large international connection. The new complex is designed as a management center, production and dissemination of knowledge.

Keywords: architecture - education - identity - modernity - space.

Resumo: O objetivo deste trabalho é analisar três momentos na história da educação pública na Argentina nos quais se produziram mudanças nas formas de entender ao estudante e o seu contexto educativo como uma experiência transformadora.

A identidade nacional está conformada pelas instituições, e a educação, desde a escola até a universidade, tem um lugar central nesta construção.

1. Desde que Argentina declarou sua independência, e, sobretudo, quando Buenos Aires se converteu na capital da nova república em 1880, começou um vertiginoso processo de modernização. Este projeto envolveu aos imigrantes que chegaram de diferentes paises à capital e a transformaram numa Babel que o governo teve que "argentinizar". Assim, a educação pública obrigatória, gratuita e laica foi regulamentada em 1884 com a aprovação da lei 1420. As escolas públicas abasteceram o novo espaço onde os estudantes puderam obter os valores nacionais.

2. Durante o primeiro peronismo (1943-1955) a educação técnica teve um papel central dentro do projeto modernizador que no discurso governamental se apresentava como revolucionário: o da Nova Argentina, onde os trabalhadores ocuparam um lugar privilegiado. Neste período se criaram diversas tipologias: desde a escola-fábrica até a Universidade dos Trabalhadores na que, através de um novo sistema de administração, o espaço escolar oferecia serviços à comunidade que superavam a função educativa tradicional. 
3. Atualmente, o sítio onde havia uma antiga bodega, numa das zonas mais cotizadas de Buenos Aires, se desenvolveu um projeto educativo: o Pólo científico e tecnológico, dividido em diferentes secções: a sede do Ministério de Ciência, Tecnologia e Inovação Produtiva, CONICET, e a Agencia Nacional de Promoção de Ciência e Tecnologia e institutos de gestão, produção e difusão do conhecimento.

Palavras chave: arquitetura - educação - espaço - identidade - modernidade. 